\title{
Sistemas de riego en la zona de Chiltazon-La Concepción de los siglos XVI al XVIII
}

\author{
Dayuma Guayasamín Ortíz
}

Resumen

El PRESENTE ARTículo TRATA SOBRE LA RELACIÓN DEL SER HUMANO CON LA NATURALEZA, EN DOS ÉPOCAS DISTINTAS en la provincia del Carchi al Norte del Ecuador: la época Precolombina y la época de las haciendas. LOS SISTEMAS DE RIEGO HAN SIDO HERRAMIENTAS DE CONTROL SOBRE LA NATURALEZA POR PARTE DE LOS GRUPOS HUMANOS, SIENDO ARTICULADORES DE SISTEMAS ECONÓMICOS, POLÍTICOS Y SOCIALES DIFERENTES. EN ÉPOCA DE LOS

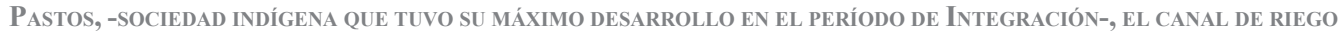
TIPUYA SE ESTABLECIÓ COMO PARTE DE UN SISTEMA ECONÓMICO MICROVERTICAL, QUE PERMITIÓ EL APROVECHAMIENTO

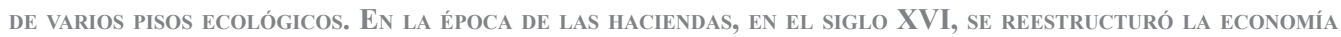
DE LA REGIÓN, CAMBIANDO DE ESTE MODO LOS SISTEMAS AGRÍCOLAS INDÍGENAS POR PLANTACIONES IMPUESTAS POR EL SISTEMA COLONIAL, QUE REUTILIZARON ESTOS SISTEMAS DE RIEGO, EN ESPECIAL EL CANAL TIPUYA.

Palabras Clave: pastos - arqueología - Carchi - sistemas de riego - tipuya.

Abstract

The ARTICLE DEALS WITH THE RELATIONSHIP BETWEEN HUMAN BEINGS AND NATURE IN THE PROVINCE OF CARCHI IN Northern Ecuador during the Precolombian and haciendas eras.

IRRIGATION SYSTEMS AS A TOOL OF ENVIRONMENTAL CONTROL ALSO REPRESENTED A JUNCTION FOR DIFFERENT eConomic, political and social systems, to interact. In the time of the Pastos, an indigenous society

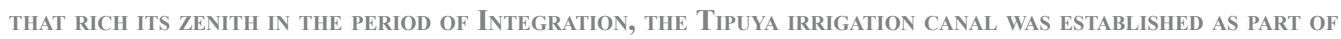
A MICRO-VERTICAL ECONOMIC SYSTEM, THAT ALLOWED THE EXPLOITATION OF VARIOUS ECOLOGICAL ZONES. IN THE ERA OF HACIENDAS, IN THE SIXTEENTH CENTURY, THE ECONOMY OF THE REGION WAS RESTRUCTURED REPLACING THE INDIGENOUS AGRICULTURAL SYSTEMS WITH PLANTATIONS IMPOSED BY THE COLONIAL SYSTEM THAT TOOK ADVANTAGE OF THESE IRRIGATION SYSTEMS, ESPECIALlY THE TIPUYa CANAL.

KEY WORDS: PASTOS- ARQUEOLOGY - CARCHI - IRRIGATION SYSTEMS - TIPUYA.

1 Este artículo es una síntesis de la disertación de tesis presentada para la obtención de la licenciatura en Antropología con mención en Arqueología en la Universidad Católica del Ecuador. La autora ha trabajado en varias proyectos de investigación en la Costa, Sierra y Oriente, con experiencia en trabajo de campo y laboratorio. Actualmente desempeña el cargo de arqueóloga regional en el Instituto Nacional de Patrimonio Cultural, Regional Cuenca. 


\section{Introducción}

1 estudio de los sistemas de riego en la zona de Chiltazón - La Concepción del Siglo
XVI al XVIII, trata sobre la economía política de una región del Carchi, ubicada al
Norte del Ecuador, tanto en la época prehispánica y durante las haciendas. Existen en la zona varios canales que denotan gran antigüedad, pudiendo tratarse de construcciones realizadas en épocas precolombinas. Los Pastos son una sociedad que, según Uribe (1977), estuvo presente desde el siglo VIII hasta la época de la conquista española en 1530 en la actual provincia del Carchi y en el departamento de Nariño, en Colombia. Autores como De Francisco (1971) establecieron que esta sociedad tuvo tres fases culturales: Capulí, Piartal y Tuza. Uno de los sistemas de riego en la región de estudio es el sistema Tipuya, el cual será analizado como un articulador de estos dos tipos de economía desde varias entradas.

El estudio propuso cuatro objetivos en torno a este sistema de riego. El primero fue reconstruir geográficamente el sistema de riego Tipuya. Esto se realizó a través del mapeo del canal para establecer las fuentes de captación y las zonas irrigadas, analizando la técnica de construcción así como los materiales utilizados y los cambios durante el período de estudio. El segundo objetivo intentó estudiar las interacciones políticas, sociales y económicas de las sociedades que utilizaron este sistema de riego. El tercer objetivo estableció los sistemas agrícolas relacionados con los sistemas de riego para observar las diversas formas de modificaciones del espacio implementadas por los Pastos. Finalmente, el cuarto objetivo fue la realización del estudio del sistema de hacienda, de las formas de utilización de los canales y de los nuevos sistemas agrícolas que se introducen en esta época.

En lo que respecta a la época de construcción y la sociedad responsable de esto, la primera hipótesis que surge es que existía un sistema de riego con varios canales que tienen sus fuentes en los páramos de Chiltazón y Chulte y que terminan irrigando las zonas bajas de La ConcepciónSanta Ana, en el valle del río Mira. La construcción de este sistema hidráulico fue hecha por una sociedad prehispánica, que planificó el trayecto del agua. Una de las sociedades que habitaron esta zona fue la cultura Pasto, la cual podría haber construido esta obra.

Así mismo, en lo que corresponde a la época de la Colonia, el sistema de hacienda posiblemente terminó con el sistema microvertical utilizado por los grupos Pasto al darse un cambio drástico en el paisaje agrario. Sin embargo, los canales de agua de la zona de ChiltazónLa Concepción presentan modificaciones en su estructura constructiva, lo que lleva a suponer que fueron reutilizados por el sistema de hacienda que se desarrolló en la zona.

Finalmente, es importante poner en evidencia que, con la llegada del sistema hacendatario al Carchi en el siglo XVI, se puso en marcha una reestructuración de la economía en la región. Este nuevo sistema cambió las relaciones laborales, políticas y económicas entre las poblaciones asentadas en ese lugar e incluso transformó el sistema agrícola nativo. Lo cual llevaría a pensar que en las partes altas, se habría desplazado el cultivo de papa, quinua y maíz a favor de una economía más bien ganadera; mientras que, en las partes bajas, los cultivos de coca, ají y algodón podrían haber sido remplazados por plantaciones mayoritariamente de caña de azúcar.

\section{Lineamientos teóricos}

Para empezar, es importante mencionar que la teoría que ha propuesto John Murra (1975) sobre el control de los pisos ecológicos, basada en ejemplos de la sociedad Inca en el Perú, sirvió de base para este análisis. En efecto, este sistema económico de un control máximo de pisos altitudinales por parte de las sociedades andinas de la Puna es un modelo vertical que se daba en todos los Andes, debido a una percepción similar de la obtención de los recursos. Esto significa 
que se intentó controlar la mayor cantidad de pisos ecológicos por una misma sociedad para la obtención de un número mayor de productos destinados a la supervivencia y al intercambio.

\begin{abstract}
"Cada grupo étnico [...] trataba de controlar y abarcar con la gente a su disposición la mayor cantidad de pisos ecológicos. Grandes o pequeños, los grupos étnicos tenían una percepción similar de los recursos y la manera de obtenerlos. El deseo de controlar zonas climáticas alejadas mediante colonos permanentes, determinó un patrón de asentamiento y de control vertical cuya distribución fue probablemente panandina". (Murra, 1975: 50).
\end{abstract}

Esta teoría tiene relación con un control económico simultáneo por parte de las etnias locales sobre diferentes "islas ecológicas" situadas a grandes distancias del centro de poder en dónde se concentraba la población y el mando político.

En efecto, cada piso altitudinal posee un microclima diferente que permite que crezcan ciertos tipos de plantas mientras que en otros, las condiciones favorecen diferentes plantaciones. Dado que se necesitaba cultivar estos productos de forma constante, se crearon sistemas de archipiélagos en estos pisos para abarcar una mayor área de producción.

Otro autor relacionado al análisis de los sistemas de archipiélagos en la Sierra ecuatoriana y que ha estudiado el caso de los Pastos es Frank Salomon. Este autor advierte que la presencia Inca en regiones como el norte del Ecuador, si bien no duró mucho tiempo, tampoco modificó las prácticas culturales de la zona.

Udo Oberem, por su parte (1981), elabora una teoría vinculada a los Andes de páramo. En efecto, postula que en estas zonas se dio la "Microverticalidad" que se refiere a:

"Un fenómeno que [...] se distingue de la "macro" verticalidad descrita por John V. Murra (1975) por la falta de las "islas habitadas" que caracteriza el sistema archipiélago como tal. Microverticalidad quiere decir que los habitantes de un pueblo tenían campos situados en diferentes pisos ecológicos alcanzables en un mismo día con la posibilidad de regresar al lugar de residencia por la noche". (Ibíd.: 51)

Relacionando estos autores con otros, Wittfogel, en el caso de que un número de cultivadores quiera conquistar valles y tierras áridas para la producción de cierto tipo de bien agrícola, se ven forzados a congregarse en grupos organizacionales, en los cuales tendrán que trabajar en cooperación con los demás miembros de la sociedad que hagan uso de los sistemas de riego en su libro, Despotismo oriental (1966), habla de sociedades que empiezan a adquirir poder sobre otras gracias al control de los sistemas de irrigación, modificando su sistema tanto político como económico.

Según Wittfogel (1966), las sociedades, al responder a causas históricas ya sean estas simples o complejas, tienen que transformar su medio ambiente y es en este intento de transformación que se desarrolla un cuerpo técnico que va a alterar su organización social y la manera en que van a percibir el mundo. El medio ambiente es por lo tanto un factor que condiciona el uso de cierto tipo de tecnología provocando cambios sociales. De esta forma, las condiciones históricas específicas antes mencionadas permiten que se actualice o se cree un cierto tipo de potencial hidráulico.

Como bien nos dice Wittfogel, “...en el caso de que un número de cultivadores quiera conquistar valles y tierras áridas para la producción de cierto tipo de bien agrícola, se ven forzados a congregarse en grupos organizacionales, en los cuales tendrán que trabajar en cooperación con los demás miembros de la sociedad que hagan uso de los sistemas de riego" (Ibíd.: 37). Pero lo que hay que resaltar es que esta organización está controlada por una autoridad a la cual se subordinan los demás, ya que es la encargada de manejar este recurso y de distribuirlo a los diferentes cultivos.

Así mismo, Gregory Knapp (1992) trata sobre los sistemas de riego específicamente y ayuda a reconocer los canales prehispánicos a través de algunas características. Se vincula con el autor 
anterior en el hecho de que describe ciertos canales que pertenecen a sociedades complejas, las cuales han modificado su organización social para poder controlar y manejar un sistema de riego. Este autor ha analizado sobre todo canales en la región norte del Ecuador.

Por otra parte, como se sabe, cada época es caracterizada por una tecnología específica de acuerdo a la disponibilidad de materiales de construcción, lo que puede ayudar a su reconocimiento temporal:

\footnotetext{
"Un virtual canal prehispánico debe ser de un material de construcción tradicional; su curso debe estar relacionado con monumentos prehistóricos como tolas (montículos) de tierra para plataformas de casa; deben estar presente cerámicas prehistóricas a lo largo del canal y dentro del área irrigada; el área irrigada debería mostrar tolas y ser inhóspita para la ocupación humana sin el riego; y se debe encontrar documentación histórica sobre la antigüedad del canal” (Ibíd.: 18).
}

Es así que un canal no debe ser tomado en cuenta por sí solo, sino que éste necesita estar en un cierto contexto etnohistórico y arqueológico para poder establecer su período de construcción.

Este trabajo fue realizado a partir de un componente etnohistórico, donde los documentos históricos revelaron evidencia sobre la economía colonial a través del sistema de hacienda en la región. En cuanto a la parte arqueológica, uno de los objetivos principales de este trabajo ha sido descubrir quiénes fueron los responsables de la construcción del sistema de riego Tipuya, razón por la cual se realizó primeramente un estudio arqueológico de la zona de estudio para tratar de ubicar los asentamientos Pastos y su distribución. El segundo paso fue encontrar un terreno al lado del canal que tuviese material cultural con la intención de probar que Tipuya pudo haber estado relacionado con la cultura Pasto. Para esto, se hizo un análisis cerámico del material encontrado para compararlo con estudios hechos anteriormente por autores como De Francisco (1971) y Echeverría (1994).

\section{Historia y geografía de la zona}

La cultura Pasto se asentó en la actual provincia del Carchi al norte del Ecuador en dónde los ríos Carchi y San Juan sirven de frontera con Colombia, al sur limita con la provincia de Imbabura, al este con la provincia de Sucumbios, y a occidente con la de Esmeraldas. Dentro de esta provincia, el área de estudio es delimitado por el sistema de riego Tipuya. Esto significa que se tomará en cuenta la zona desde la bocatoma hasta las zonas irrigadas, es decir desde el cruce de los ríos San Juan y el Rosario hasta el valle del Chota-Mira, específicamente la zonas de La Concepción y Santa Ana.

Este gráfico presenta los sitios por donde pasa el sistema de riego Tipuya y en los cuales se realizaron los respectivos trabajos arqueológicos. Dentro de los principales lugares se encuentran Palo Blanco, los terrenos de la Sra. Bolaños, Arrayán Loma y La Concepción-Santa Ana. Igualmente está representado el canal desde la bocatoma hasta los campos de riego ubicados en el valle del Chota-Mira.

Por un lado, tenemos a las zonas altas que corresponden a los Andes de páramo y por el otro lado tenemos a las zonas bajas que corresponden a los valles interandinos, es decir al valle del Chota-Mira, en este caso de estudio.

Los grupos Pastos estuvieron asentados en zonas alto-andinas así como en los valles interandinos. Posiblemente los núcleos de población estaban ubicados en las zonas altas mientras que en las riberas del Chota-Mira estaban asentadas las colonias encargadas de proveer productos como el ají y la coca a los asentamientos de la sierra. Sin embargo, se sabe que existieron en esta parte del valle, varios grupos pertenecientes a diferentes cacicazgos que tuvieron el mismo objetivo, el de cultivar los extensos campos de coca.

Como se mencionó anteriormente, en las riberas del río Chota-Mira, varias colonias Pastos se asentaron con el objetivo de cultivar productos exóticos. Pero estas tierras fueron compartidas 


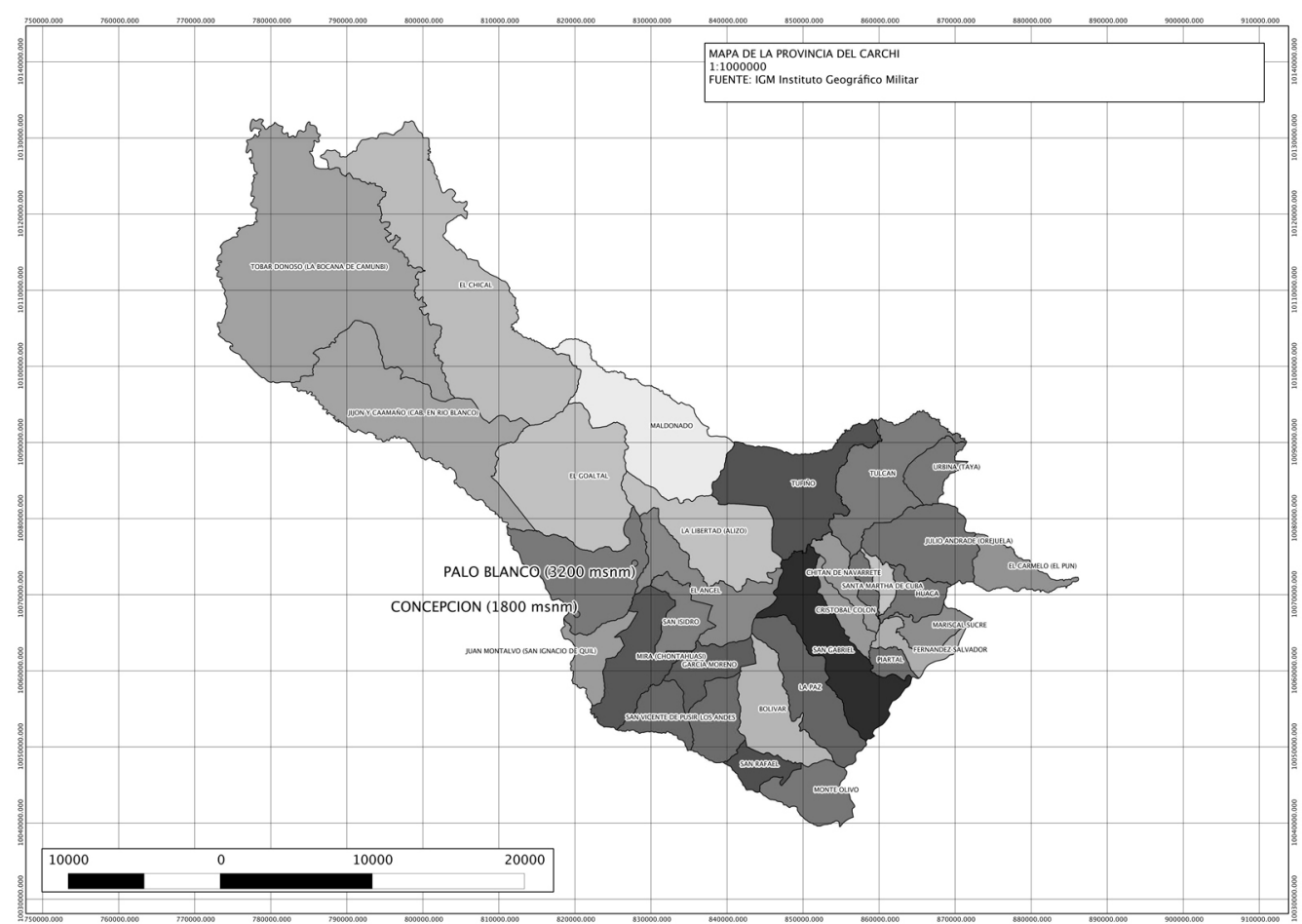

Mapa 1. Mapa regional de la provincia del Carchi

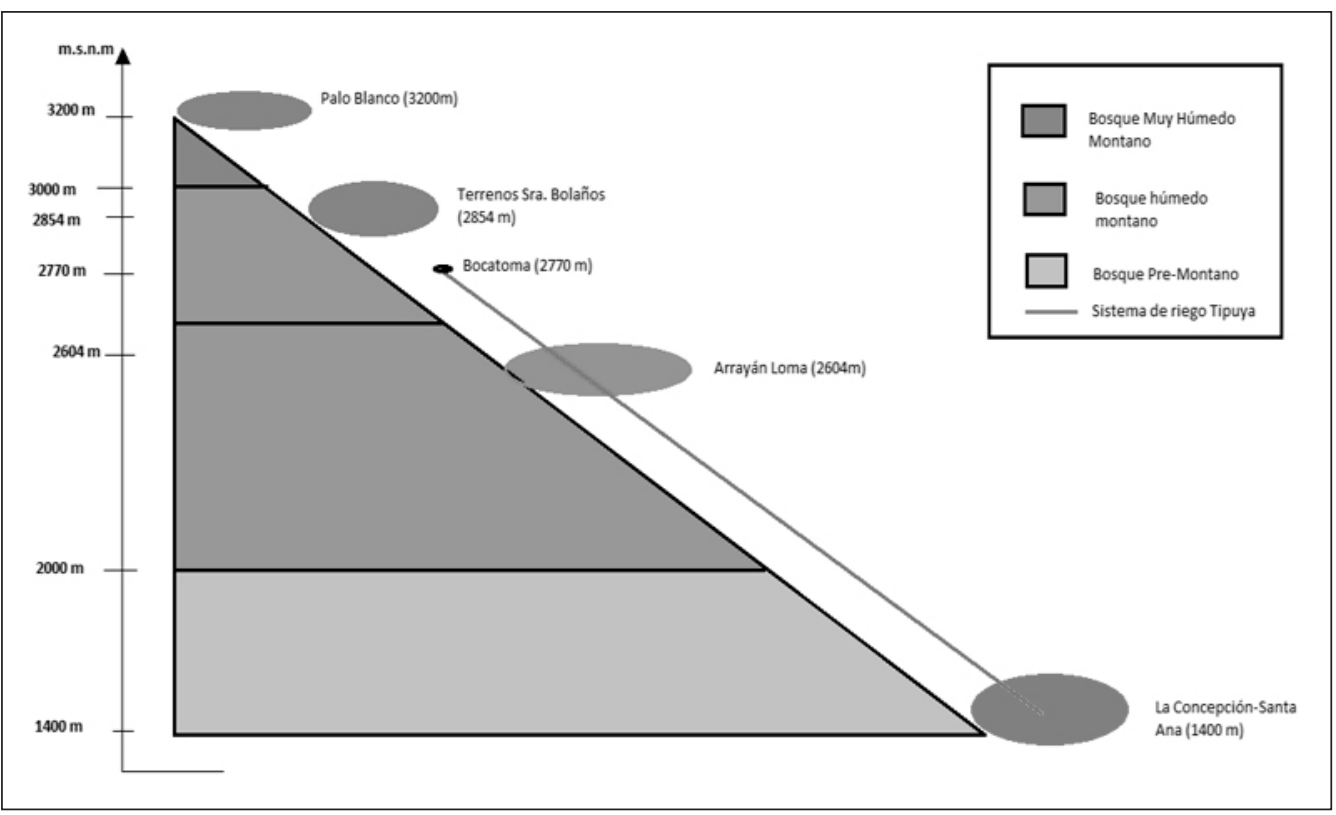

Gráfico 1. Sitios y pisos ecológicos relacionados al sistema de riego Tipuya 
por otros grupos con el mismo motivo, así como lo afirma Murra (1975). Se habla por lo tanto de islas multiétnicas en donde varias culturas compartieron el mismo territorio pero sin perder su filiación cultural.

En el caso del valle del Chota-Mira, los Lachas, los Caranquis y los Pastos ocuparon este territorio ya que eran los vecinos directos de estas tierras: Los Pastos al Norte, los Lachas al Este y los Caranquis al Sur. Aunque como lo afirma Landázuri (1990: 27), “.... a su vez los espacios de producción, como los asentamientos, fueron ocupados por varios cacicazgos de la misma etnia (multicacical), y por otra parte, fueron multiétnicos”. Esto posiblemente favoreció el trueque, intercambio y mercado.

Las fuentes del siglo XVI coinciden en afirmar que el territorio Pasto se extendió desde Colombia hasta Ecuador. Es decir, desde el departamento de Nariño hasta la actual provincia del Carchi respectivamente, limitando su territorio, al norte con los Quillacingas y al sur con los Caranquis. Los principales asentamientos definidos por los investigadores son Tulcán, Guaca, Tuza y Mira, cada una con sus respectivas parcialidades. Si bien los grupos Pastos tenían un patrón de asentamiento disperso y sus poblados eran autónomos, su organización socio-política era la de un cacicazgo. Esto evidencia una cierta complejidad política, demostrando por lo tanto que existían estructuras internas de jerarquía. Existía una autoridad conocida como Cacique el cual estaba encargado de repartir los recursos entre toda la población. El pueblo estaba obligado a entregar impuestos que podían ser comida, materia prima, productos suntuarios y de valor exótico, etc. Todo esto iba donde el Cacique, quién redistribuía estos recursos entre el pueblo. Los Incas incursionaron de modo leve en territorios Pastos, lo que permite afirmar que la influencia incaica no tuvo mayor peso y se conservaron los sistemas políticos y económicos de esta cultura. Con la llegada de los españoles, su sistema cambió drásticamente y se impuso otra manera de explotación del medio ambiente.

En lo que respecta la cronología Pasto, la periodización cultural en el Carchi aún es sujeta a varias propuestas entre las cuales constan autores como Jijón y Caamaño, Max Uhle, Echeverría; sin embargo, la más aceptada es la que define la fase Capulí (800 a $1500 \mathrm{~d}$. C) como la más antigua, seguida de la fase Piartal (750 a $1250 \mathrm{~d}$. C) y finalmente la fase Tuza (1250 a $1500 \mathrm{~d}$. C).

En lo que respecta la fase Capulí, es importante mencionar que es en este estilo cerámico donde aparecen los coqueros, figuras antropomorfas sentadas representando hombres masticando hoja de coca (protuberancias en las mejillas para hacer alusión al bollo de coca), así como las compoteras con pedestal medio y alto que son exclusivas de este grupo, las ollas pequeñas con cuatro protuberancias en los hombros, ollas globulares con formas compuestas de estrella y formas antropomorfas y zoomorfas, entre las más conocidas. Sin embargo, lo que distingue a la cerámica Capulí del resto de estilos cerámicos es la técnica de decorado conocida como Negativo, la cual se basa en cubrir la vasija, en ciertos lugares, a través de materiales impermeables como ciertas resinas para protegerla de la aplicación posterior de un color más oscuro encima, y así obtener las figuras deseadas del color de la vasija luego de retirar estos materiales. Estos diseños son exclusivamente geométricos, destacándose los círculos, las líneas, los puntos, los triángulos y los rombos.

En la fase Piartal, las formas más características de este estilo son:

"Las botijuelas de cuello recto y largo, base cónica; cántaros antropomorfos (personaje sentado en cuclillas); compoteras de pie bajo y cuerpo angular; cuencos con base anular y cuencos de recipiente cuadrangular y base anular; ocarinas (imitación del caracol marino)" (Echeverría, 2004).

En cuanto al estilo de decoración, éste se caracteriza por la utilización de una arcilla limpia en donde se observa una decoración mixta, es decir pintura roja realzando las figuras geométricas hechas en negativo. También, se realza la pintura interna de los platos de base anular, contrastando el fondo con los diseños geométricos y zoomorfos de los dibujos. En efecto, podemos encontrar en los platos figuras como rombos, círculos, triángulos, estrellas, cuadrados en lo que respecta a 
las combinaciones de figuras geométricas mientras que también se encuentran dibujados varios tipos de mono con o sin cara.

Por su parte, la cerámica Tuza es particular ya que se caracteriza por una pasta de color castaño claro y homogéneo, mientras que el desgrasante fue el cuarzo así como partículas de pirita. En esta fase aparecen los jarros de cuello alto y decorados con formas geométricas, distintivos de esta fase. En cuanto a la técnica de decoración se puede evidenciar la pintura positiva: rojo sobre crema pulido. Las representaciones que se pueden observar en los platos son figuras zoomorfas y antropomorfas dibujadas frente a frente, separadas por una línea en el medio del plato. La diversidad de figuras es amplia: caciques con sus bastones de mando así como adornos especiales en la cabeza, guerreros danzando, mercaderes, pescadores, cazadores, hombres cogidos de la mano alrededor del plato, shamanes, y representaciones de animales tanto hembras como machos de la misma especie, venados, loros, cigüeñas, cóndor, monos, jaguares, arañas y serpientes, etc. Pero quizá lo que caracteriza a este estilo son las representaciones del sol con ocho puntas dibujadas en el centro de los platos.

Los Pastos aprovecharon un máximo de pisos ecológicos para obtener productos propios de cada zona, tanto para la subsistencia como para el comercio.

Esta sociedad estuvo compuesta por cuatro cacicazgos, en la zona del Ecuador, ubicados en regiones con climas diferentes con el objetivo de abastecerse de productos de primera necesidad, por lo que crearon colonias, las cuales los proveían de estos recursos básicos. En las partes altas, los tubérculos consistieron en el producto por excelencia para cubrir las necesidades de la población, mientras que en zonas templadas hay evidencia del cultivo de maíz. En los valles interandinos, en este caso el valle del Chota-Mira, se cosechaba el algodón, el ají y la coca, productos exóticos destinados al comercio.

Es necesario centrarse en el estudio de caso, es decir sobre el sistema de riego Tipuya, el cual representa el foco de este análisis, planteado como el articulador de una economía microvertical en épocas precolombinas y como una fuente importante de riego en época de las haciendas. Razón por la cual se han planteado dos hipótesis: que Tipuya fue un canal prehispánico y que durante la colonia fue reutilizado por las haciendas instaladas en la zona.

\section{Descripción geográfica del sistema de riego Tipuya}

Como primer paso para la realización de este trabajo, se procedió, a través de la información proporcionada por gente de la comunidad de Palo Blanco, situada a 3200 m.s.n.m., a ubicar el canal de riego Tipuya, del cual nadie tenía fecha exacta de construcción. Hay que precisar que la geografía del lugar no es la de altas montañas con picos elevados, sino más bien largos macizos que son caracterizados por un sistema ecológico de páramo andino en la parte superior y valles calientes en las partes bajas en medio de las montañas, que proporcionan vastas áreas, ideales para el cultivo. Por lo que la geografía dentro de la cual se encuentra el canal de riego Tipuya, corresponde en principio a la geografía dentro de la cual se elaboran las teorías de Murra (1975), para Perú, y en el caso de Ecuador, de Oberem (1981) y Salomon (1990).

El canal de riego Tipuya, tiene su fuente de captación en el río El Rosario, lugar en donde se ha construido la bocatoma a 2770 m.s.n.m. Este canal excavado en la superficie natural (de tierra), que en algunos casos se le ha dado recubrimiento de piedra o de cemento en la actualidad, evidencia de recientes intervenciones, canaliza el agua a campos de cultivo situados en zonas bajas (aproximadamente a unos 1800 m.s.n.m.). Según el proyecto INERHI-ORSTOM (1992: 19010), el largo, en línea recta, es de 11,7 Km y su caudal es de 103 litros por segundo. El canal de riego Tipuya, que desciende del páramo de Chiltazón hasta La Concepción, atraviesa zonas con fuertes pendientes así como zonas planas. Existe una relación directa entre las áreas con declives poco o casi nada pronunciados, en donde se pudieron haber establecido asentamientos y zonas de cultivo. Estas planicies, al lado oriental del canal, en su mayoría, aprovechaban el 
hecho de que el agua corre paralela a la superficie y de esta forma se vuelve más manejable y se la puede controlar más fácilmente.

En el recorrido del canal, se tomaron 23 puntos GPS, desde la propiedad de la Sra. Rosa Bolaños (2854 m.s.n.m.), en donde se encontraron hileras de piedras a manera de pequeñas terrazas y material cerámico, hasta un reservorio arriba de La Convalecencia (1973 m.s.n.m.), en donde se encontró que el canal se dividía en dos canales secundarios para abastecer de agua a La Concepción y Santa Ana.

\section{Trabajo de campo en Arrayán Loma}

El siguiente paso, en lo que atañe el aspecto arqueológico para probar la filiación Pasto, fue encontrar cerámica de esta cultura en las áreas que recorrió el canal para de esta manera vincularlo a la utilización por parte de estas sociedades en su sistema agrícola. Con la posible evidencia cultural que se encontró se podría hablar de un grupo humano que estuvo presente en el sitio, si bien viviendo permanentemente, sino con visitas regulares al terreno en donde se cultivaba. Por lo que, fue necesario buscar un terreno al lado del canal.

La primera actividad realizada fue la georeferenciación de Arrayán Loma para conocer de manera exacta su superficie y las características del terreno en donde se realizaron las pruebas de pala. Este trabajo se lo hizo con un GPS de precisión, con el que se captó los puntos necesarios del contorno del terreno.

Los puntos GPS fueron tomados en los contornos del terreno, cada 10 metros y en donde se observaba alguna curva o los límites hasta la cual la vegetación permitía entrar. En la página previa se presentan dos gráficos de la georrefenciación del terreno de Arrayán Loma. El uno consiste en las zonas 1,2 y 3 y el segundo mapa muestra la zona 4 (ver gráfico 2 y 3 ).

El objetivo de este trabajo consistió en la recolección de material cultural y el registro de restos arquitectónicos, de asentamientos o alguna evidencia cultural junto al canal que permitiera probar el uso de éste por parte de los grupos humanos asentados en la región de estudio.

Para esto, se dividió Arrayán Loma en cuatro zonas para poder ubicarlas de mejor manera en el caso de que aparezca material. Hay que precisar que Tipuya pasa por los puntos 1 y 16 (ver gráfico 2). Las tres primeras zonas pertenecen a terrenos del Sr. Urpiano Guerrero. La zona 1 se encuentra en la parte superior Norte del canal y presenta una pendiente más o menos pronunciada con ciertos cortes a manera de terrazas. La zona 2 se encuentra en la parte inferior del canal, justo donde está construida la casa del dueño del terreno, así como un camino hecho para que entren vehículos, por lo que se puede decir que esta zona es la más disturbada. La zona 3 corresponde al terreno que linda con el del otro dueño, separados éstos por una cerca. Finalmente, la zona 4, ubicada en dirección Suroeste, corresponde al terreno perteneciente al Sr. Luis López (Ver Gráfico 3). En cada zona se hicieron los transectos separados por 5 metros y llamándolos por orden alfabético, aunque como se verá más adelante, las últimas zonas fueron excavadas cada 10 metros por las condiciones del terreno. La técnica que se aplicó para este caso es el del rejillado. Se trazaron transectos paralelos en toda la superficie del sitio cada 5 metros, sentido Este para seguir las curvas de nivel del terreno, así como líneas perpendiculares. Una vez hecho el rejillado, que tuvo porciones de 5 x $5 \mathrm{~m}$, se hicieron pruebas de pala en los vértices de cada una de las rejillas, de unos 40 o $50 \mathrm{~cm}$ de profundidad y se recogió el material cultural.

Cuando se encontró material, se puso positiva a la prueba de pala, mientras que en las que no se evidenció material cultural, se puso negativas. Se excavó por transectos y en cada prueba de pala se retiró la tierra de acuerdo a los depósitos, que eran visibles con el cambio de color en la tierra. En cada uno de estos, se registró la textura, el color, la compactación y la plasticidad. En total se realizaron 156 pruebas de pala, de las cuales 152 fueron hechas para recoger material cultural, mientras que 4 se realizaron para obtener las muestras de suelo para ser analizadas y estudiar las evidencias de las actividades agrícolas. 


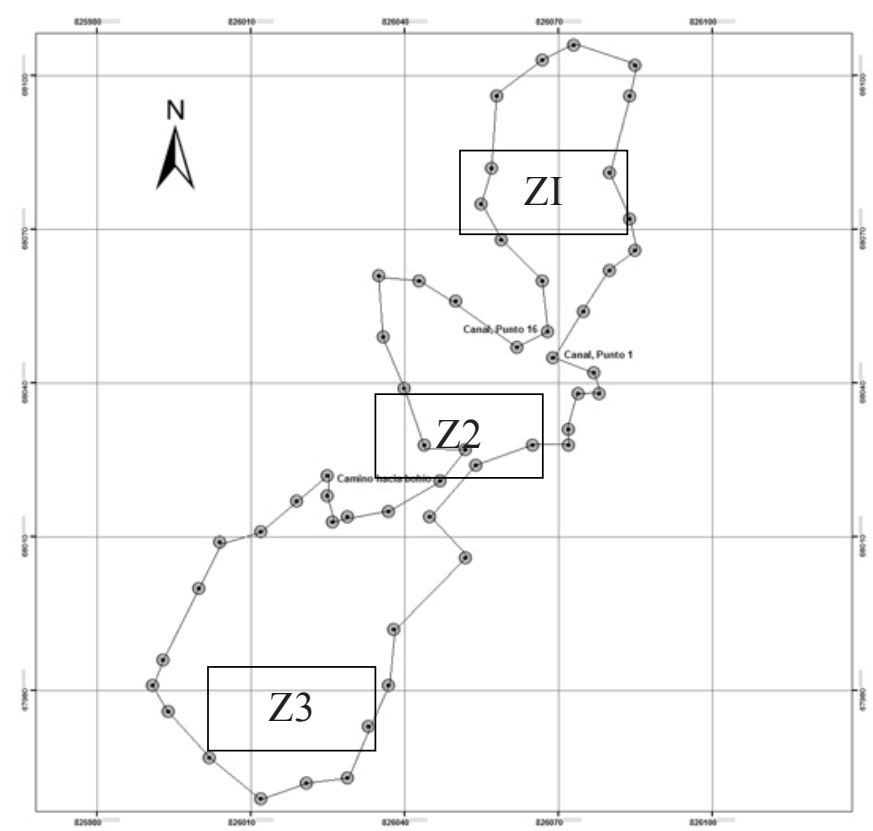

Georreferenciación del Proyecto: Sistema de Riego en la Zona de Chiltazón-Concepción del siglo $\mathrm{VI}$ al VIII.
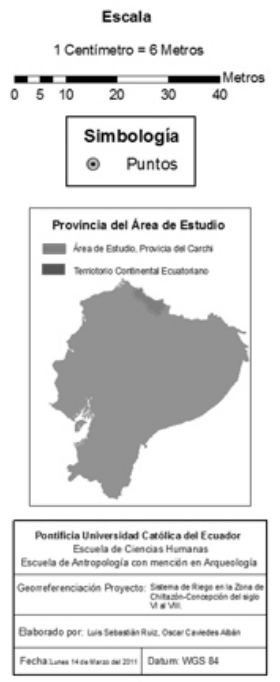

Gráfico 2. Georreferenciación de Arrayán Loma, zonas 1, 2 y 3

Arrayán Loma, es una explanada un poco más pequeña, de 2 a 3 hectáreas y con poca vegetación, lo que facilitó la prospección arqueológica.

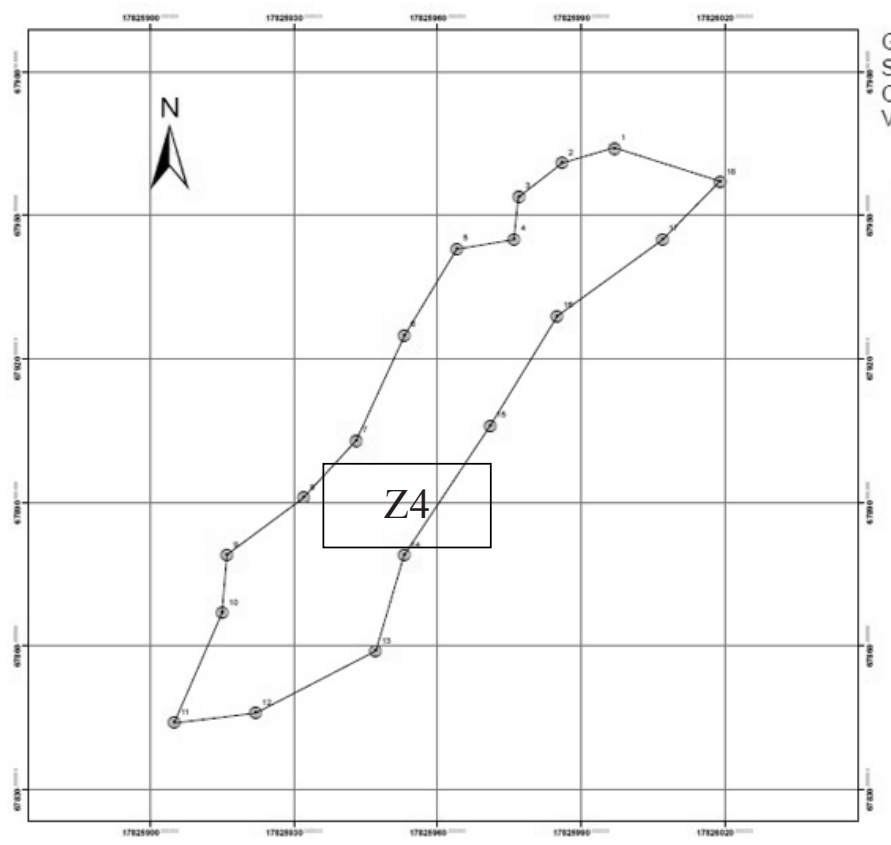

Georreferenciación del Proyecto Sistema de Riego en la Zona de Chiltazón-Concepción del siglo VI al VIII. Parte 2

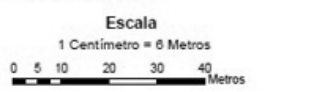

Simbologia

• Puntos
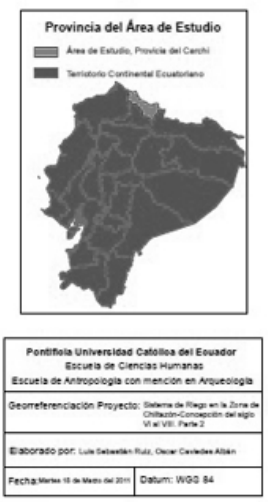

Gráfico 3. Georreferenciación de Arrayán Loma, zona 4 
Cada material cultural que fue recogido, se lo puso en una funda con su respectiva tarjeta indicando el transecto, el nivel, la profundidad, el número de procedencia, el número de funda y la fecha. Al igual que las muestras de suelo fueron recogidas sin ningún tipo de contaminación. De igual forma se hicieron cortes estratigráficos a los lados del canal para controlar los niveles en las pruebas de pala y establecer la estratigrafía así como observar las posibles capas, evidencia de limpieza del canal, en lo posible.

En resumen, de la zona 1, tres pruebas de pala dieron positivo en cuanto a evidencia arqueológica. Se pudieron obtener 8 fragmentos de cerámica, todos de tipo colonial. Éstos tiestos, vidriados de color amarillo con negro y verde son clara evidencia de la época de las haciendas en la zona, es decir evidencia de la presencia de actividad humana en Arrayán Loma en los siglos XVI a XVIII. Por otra parte, sólo se encontró este tipo de cerámica en la parte superior del canal (Zona 1), evidencia que en este período, las personas se asentaron en esa parte del terreno.

En la zona 2, que es la parte al Sur del canal y en donde está construida una pequeña choza de época reciente, se pudo encontrar un fragmento de cerámica, que presumiblemente se trataría de un fragmento de teja relacionado a la construcción de la casa. Contrariamente, en la zona 3, no se encontró evidencia de presencia humana en época prehispánica ni de la época de las haciendas. Finalmente, la zona 4, ubicada en la parte sur occidental del canal y la que se encuentra al filo de la quebrada, aparecieron varios fragmentos de cerámica, entre las cuales están pedazos de teja, posiblemente relacionados a la construcción de una casa en esta zona, destruida hace algunos años.
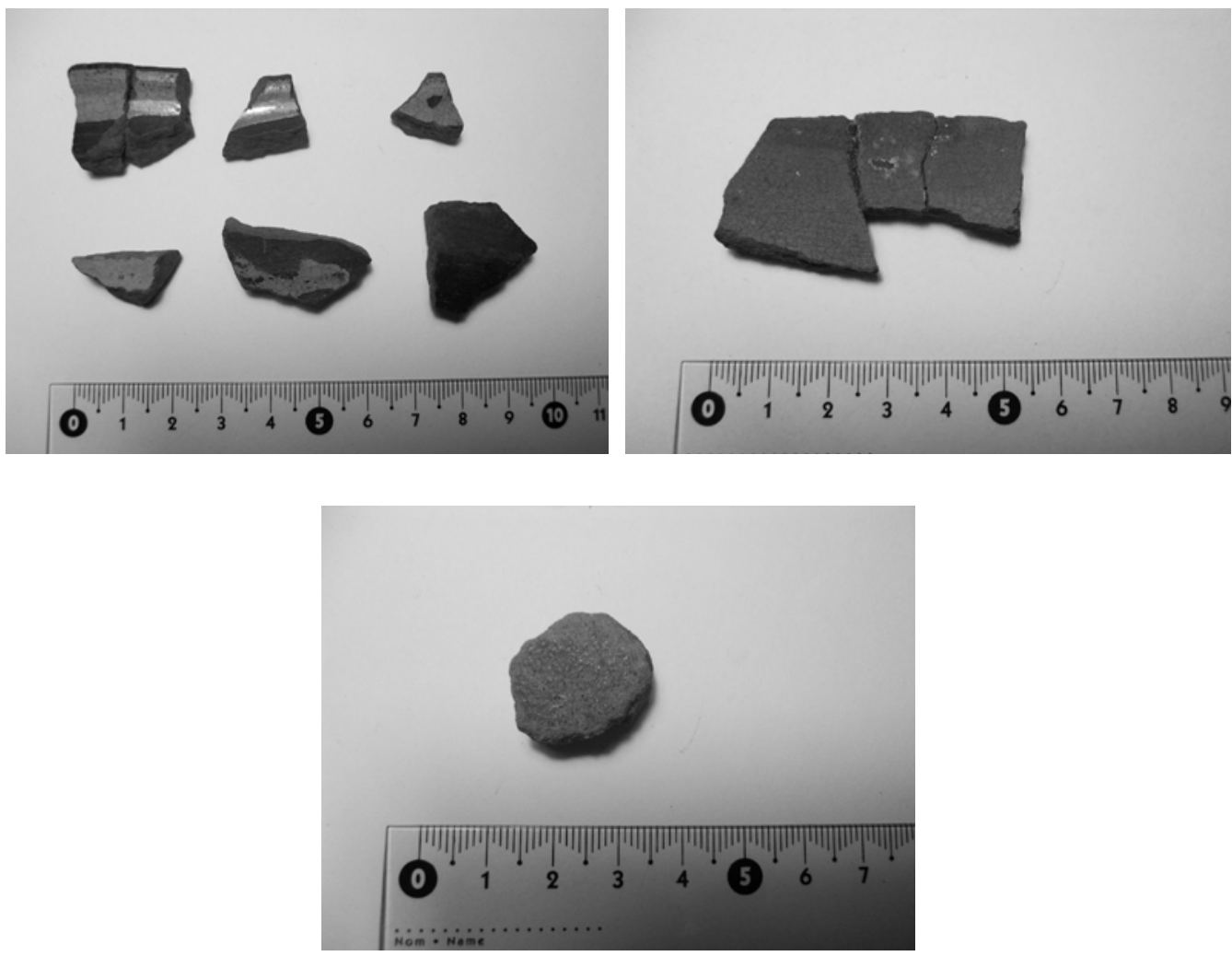

Foto 1. Fragmentos de cerámica con procedencias 1,2 y 3 de la zona 1 
Finalmente, podemos clasificar estos 18 fragmentos de cerámica en cuatro grupos distintos. El primero correspondería a la zona 1 en la cual aparecieron los fragmentos de cerámica colonial (procedencias 1, 2 y 3).El segundo grupo corresponde a los 4 fragmentos de teja (procedencias 4,6 y 7) encontrados la mayoría en la zona 4 y uno sólo en la zona 2 , cerca de la casa

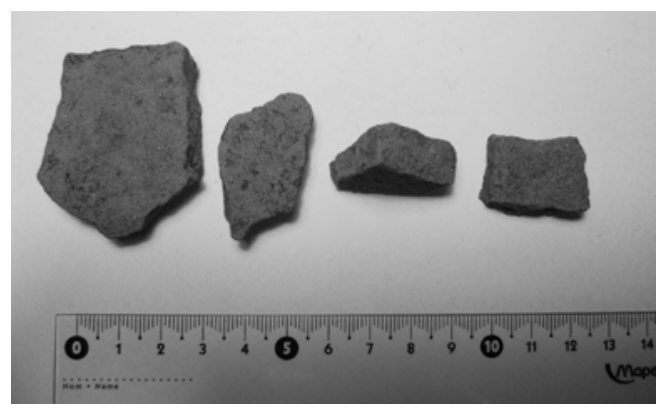

Foto 2. Fragmentos de cerámica con procedencias 4, 6 y 7 de la zona 2 y 4

El tercer grupo consta de 5 fragmentos de cerámica (procedencias 8,910 y 11) con características similares descritas arriba que podrían ser pertenecientes a cerámica Pasto.

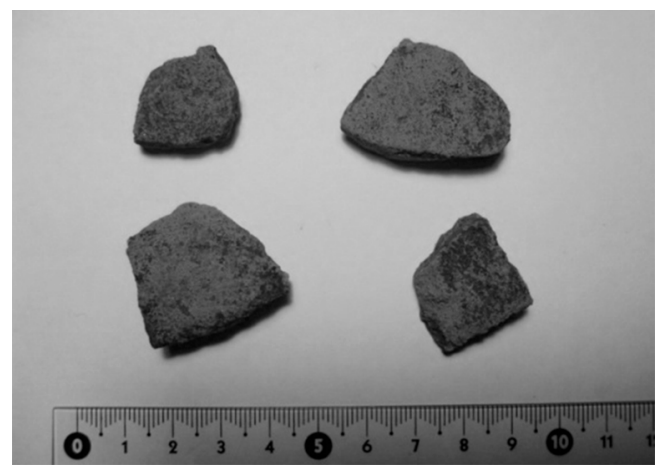

Foto 3. Fragmentos de cerámica con procedencias 8, 9, 10 y 11

Finalmente el cuarto grupo, que corresponde al tiesto con procedencia 5, se diferencia del resto por el color de la pasta, más oscura, y la abundante presencia de mica como desgrasante así como evidencia de hollín en la parte interior del tiesto.
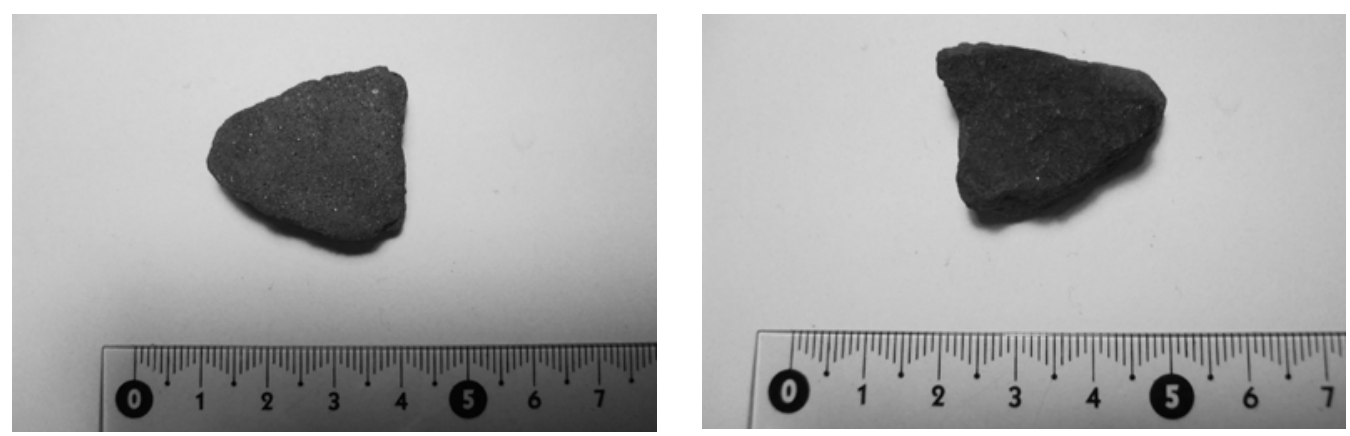

Foto 4: Fragmentos de Cerámica con procedencia 5 


\section{Trabajo de campo en los terrenos de la Sra. Bolaños}

Una vez realizado el trabajo en Arrayán Loma, era necesario buscar un sitio relativamente cerca de esta planicie con evidencia de cerámica para realizar una recolección superficial del material cultural y poder comparar con la cerámica encontrada en Arrayán Loma. De esta manera, se podría confirmar la hipótesis de que se trataba de cerámica utilizada por el mismo grupo cultural, ubicado en las partes altas, posiblemente sociedades Pasto. Los terrenos de la Sra. Bolaños se encuentran a una altura de 2854 m.s.n.m. y están ubicados a 250 m aproximadamente arriba de la bocatoma del canal Tipuya, a unos $6 \mathrm{Km}$ en línea recta de Arrayán. No es difícil imaginarse que la gente que estuvo habitando esta zona pudo haber sido responsable del control de la bocatoma en épocas de invierno y verano, así como de la limpieza del canal, dada su cercanía así como la importante fuente de agua que representaban en el río San Juan y el Rosario. En este terreno, se procedió a realizar la recolección superficial en dos zonas. Se pudo obtener en la concentración cerámica 44 fragmentos, entre bordes (6), bases (3) y fragmentos de cuerpos (33) así como dos metates. Se realizó el análisis tipológico de los 9 fragmentos de cerámica diagnóstica (bordes y bases) que se muestra a continuación.

BASE DE COMPOTERA
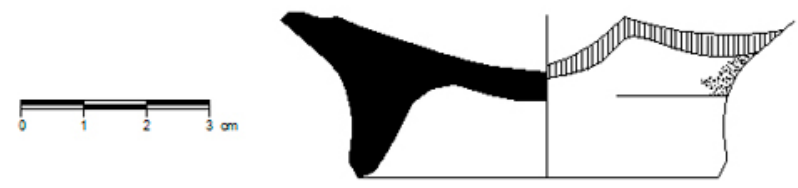

BASE DE OLLA
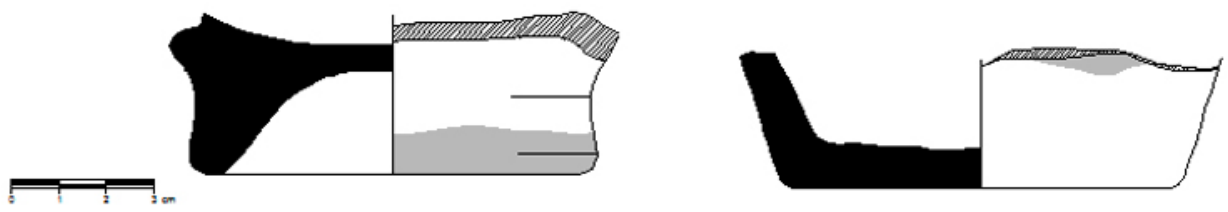

Borde DE PLLA

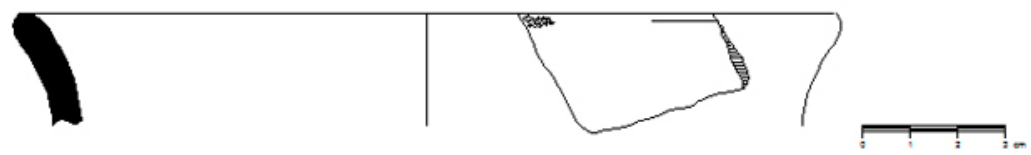



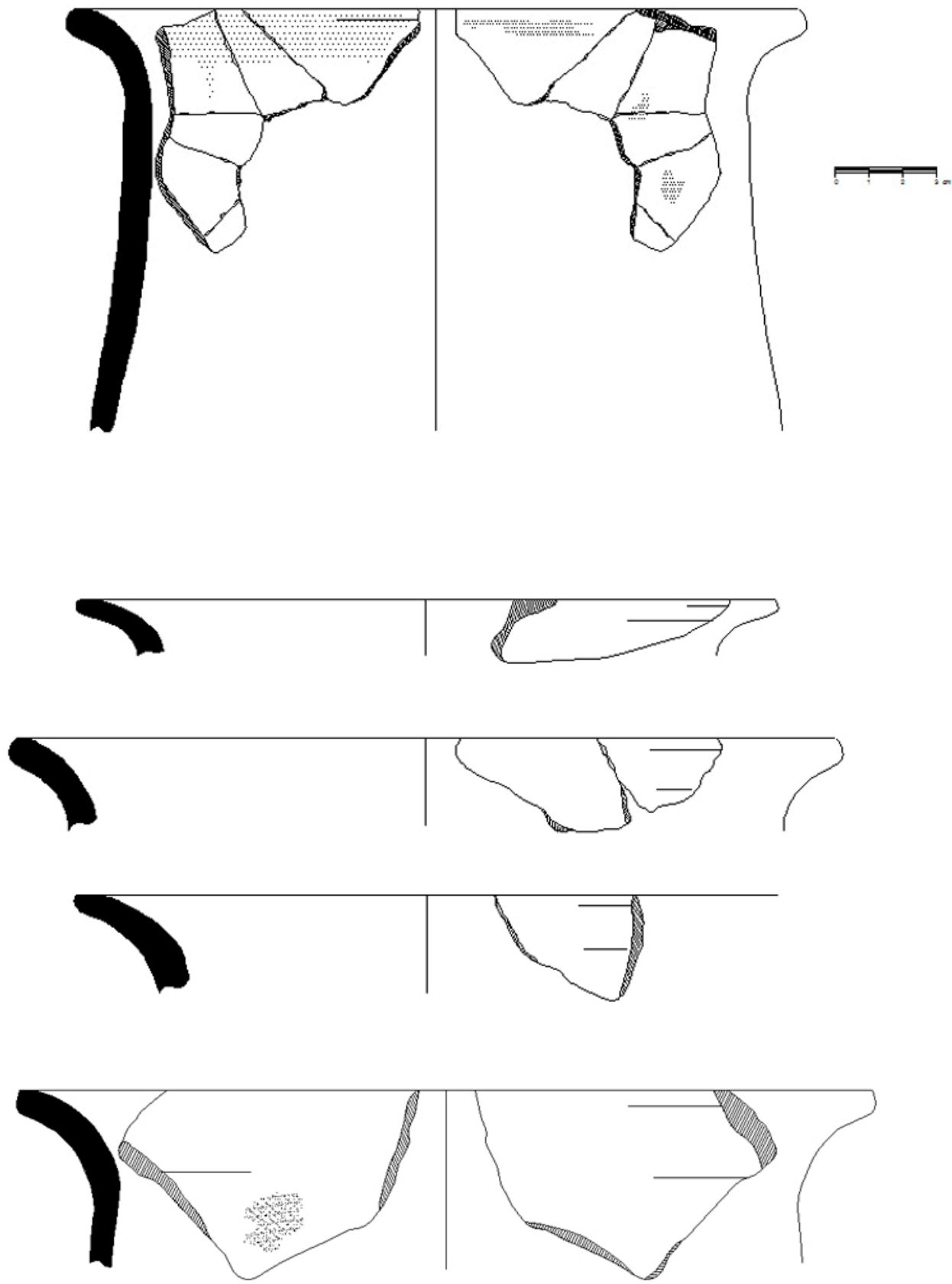
Los fragmentos cerámicos encontrados en los terrenos de la Sra. Bolaños sirven para la comparación con el material encontrado en Arrayán Loma. Por asociación al espacio geográfico en el que se encuentra, posiblemente se trate de cerámica de filiación Pasto, sin embargo es necesario compararla con cerámica de procedencia registrada para poder establecer de manera concreta que la cerámica encontrada en Arrayán Loma pertenece a asentamientos Pastos, y por ende comprobar que el canal fue utilizado por estos grupos.

La pasta de estas dos vasijas es de color café y presenta una cantidad grande de mica. En las fisuras de éstas, se puede observar que el tamaño del grano es mediano y presenta evidencias del desgrasante. Estas características de las vasijas de la reserva del Instituo Nacional de Patrimonio Cultural son muy similares sino iguales a la cerámica de Arrayán Loma y de los terrenos de arriba de la bocatoma, poniendo en evidencia entonces que se trata de cerámica Pasto.

\section{Conclusiones}

\section{Canal Precolombino}

Para este estudio es importante partir con esta cita referente a Knapp, en cuanto al análisis del canal de riego Tipuya, el cual establece enfáticamente con respecto al Valle del Chota y por ende al valle del Mira, que sino todos, casi la mayoría de los canales en el valle deben corresponder a canales prehistóricos (Knapp, 1992: 33).

Este mismo autor establece cinco parámetros que caracterizan a un canal de riego precolombino: el primero es que se trate de un canal hecho en tierra con tramos que atraviesen la roca. El segundo aspecto es su relación con sitios arqueológicos cercanos. El tercero tiene que ver con la aparición de cerámica a lo largo de éste y dentro del área irrigada. La cuarta característica enumerada por Knapp está en relación al área irrigada, la cual no puede producir sin agua. Finalmente, no hay que olvidar la documentación histórica sobre la antigüedad del canal, la cual proporciona datos interesantes que ayudan a datar a este sistema de riego en un contexto general. Después del trabajo arqueológico realizado en los terrenos y presentados aquí, se puede concluir que el canal de riego Tipuya cumple con todas las características enumeradas por Gregory Knapp, Patricia Mothes (1987) y José Echeverría, que se trata de un canal de riego Precolombino, construido en época Pasto.

\section{Canal de la Fase Tuza}

En el análisis de la pasta de los fragmentos estudiados, se puede observar que en la mayoría de ellos, el desgrasante utilizado. En efecto, se trata de pequeñas partículas blancas, claramente cuarzo. No se evidencia rastros de arena volcánica en la pasta y el color de ésta en su mayoría es café. Así mismo el nivel de oxigenación el momento de cocer es relativamente bueno dado que de los nueve elementos diagnósticos, sólo dos eran reductores mientras que los siete restantes eran oxidantes y parcialmente oxidantes. Así mismo en cuanto a la textura, se observa un alto nivel de resistencia. Todas estas características permiten suponer que se trata de una cerámica de la fase Tuza (Bastidas, 1994; De Francisco, 1971).

En lo que respecta la tipología, como se mencionó anteriormente, es un intento de clasificación de la cerámica utilitaria debido a la falta de referencias. Sin embargo, con las pocas piezas diagnósticas que se obtuvieron, se puede realizar una comparación aproximada de los bordes, labios y bases con los análisis realizados en los primeros estudios. Para esto, se utilizó la tesis doctoral de De Francisco (1971), las publicaciones de Germán Bastidas (1994), Jacinto Jijón y Caamaño (1997) así como los estudios de José Echeverría (2004). La comparación de la cerámica diagnóstico encontrada en esta tesis muestra claramente elementos característicos de cerámica relacionada con la Fase Tuza (1250 a 1500 D.C), según Uribe (1977), la fase que tuvo relación con la llegada de los españoles. 


\section{Reutilización del canal Tipuya en época de las haciendas}

Es importante partir del estudio de la estratigrafía la cual presenta evidencia de poca disturbación y la presencia de piezas cerámicas Pasto en los mismos niveles de la cerámica colonial, mostrando de esta forma la contemporaneidad cultural. Además hay que resaltar que el material utilizado por los españoles al realizar las obras de construcción, ya sea de casas como obras hidráulicas, era la piedra y la cal preferentemente. Al recorrer este sistema de riego se pudo observar algunos tramos con piedra y restos de cal, lo que indicaría una intervención en época de la colonia. Igualmente, como se presentó anteriormente, los bordes encontrados en la procedencia 1, 2 y 3 pertenecen a pequeños cuencos coloniales. Por lo tanto, el hecho de haber encontrado cerámica colonial al lado del canal de riego es muestra de que los españoles estuvieron ocupando esos terrenos para la agricultura y seguramente utilizaron el canal de riego Tipuya para sus riegos o, hubo una influencia española en la gente que aún habitaba en la zona y cerca del canal.

Por todo el análisis realizado al canal de riego Tipuya, se puede decir que éste actuó como articulador de esta economía microvertical, permitiendo la agricultura de productos básicos y exóticos en la zona del Valle del Chota-Mira. Este canal, nace en los páramos de Chiltazón y termina irrigando la zona de la Concepción, si bien durante su trayecto, hasta en épocas precolombinas, permitió irrigar igualmente terrenos ubicados a los lados. En efecto, la gente Pasto de la Fase Tuza (1250 a 1500 d.C.), en lo que al área de estudio respecta, estuvo asentada desde el sitio de Palo Blanco (ubicado en zona fría) hasta el valle, en lo que hoy se conoce como Concepción y Santa Ana. A través de las pruebas arqueológicas, se sabe que una colonia estuvo asentada en la parte superior de la bocatoma (terrenos de la Sra. Bolaños), seguramente controlando el ingreso de agua al canal y manteniéndolo en época de crecidas y verano. Así mismo, en Arrayán Loma, hubo una pequeña colonia temporal dedicada al cultivo posiblemente de maíz y algunos cereales debido a su clima templado, utilizando canales secundarios, llevando el agua a las partes planas del terreno. Finalmente, por información etnohistórica y estudios arqueológicos, se sabe que en el Valle hubo asentamientos multiétnicos (Caranquis y Pastos) que compartieron esta zona y estuvieron dedicados al cultivo de coca, ají y algodón, productos exóticos destinados al comercio así como al uso diario. Estas islas multiétnicas tenían claras normas de explotación y aprovechamiento de los recursos.

A la llegada de los españoles, las haciendas de La Concepción y Santa Ana ocuparon la zona del valle con cultivos de caña de azúcar. Reutilizaron el canal de riego Tipuya y ocuparon Arrayán Loma para sus propios cultivos. De esta forma, el sistema de riego Tipuya pasó de ser articulador de esta economía microvertical de los Pastos a ser una herramienta para este sistema de hacienda instalado en la región en época de conquista, que transformó los sistemas agrícolas establecidos desde épocas prehispánicas. Si bien su propósito fue el mismo, regar los cultivos del valle, su contexto cultural cambió drásticamente. 


\section{Bibliografía}

Bastidas Vaca, German. 1994. Exploración arqueológica del Carchi. Centro Casa de la Cultura Ecuatoriana, Núcleo del Carchi. Quito, Ecuador.

De Francisco, Alice. 1971. An Archaeological Sequence from Carchi, Ecuador. University Microfilms, Michigan, USA.

Echeverría Almeida, José. 2004 Las Sociedades Prehispánicas de la Sierra Norte del Ecuador. Una aproximación arqueológica y antropológica. Instituto Otavaleño de Antropología. Serie I, Pespectiva Histórica No.1. Quito, Ecuador.

Jijón y Caamaño, Jacinto. 1997. Antropología Prehispánica del Ecuador. Abya-Yala. Ecuador.

Knapp, Gregory. 1992. Riego Precolonial y Tradicional en la Sierra Norte del Ecuador. Hombre y Ambiente 22. Abya-Yala, Quito.

Landázuri, Cristóbal. 1990. Territorios y pueblos: la sociedad Pasto en los siglos XVI y XVII. Memorias, Volumen 1, No. 1 Marka, Quito.

1990. Pueblos indígenas de los valles interandinos: El caso de Pimampiro (siglo XVI), en Quitumbe No. 7 Revista del Departamento de Ciencias Históricas de la Universidad Católica, p. 11-35, Quito.

Mothes, Patricia. 1987. La acequia del pueblo de Pimampiro: riego tradicional en el norte del Ecuador. En Ecuador Debate, "Riego en los Andes Ecuatorianos", CAAP, No.14. Noviembre, Quito, Ecuador.

Murra, John. V. 1975 Formaciones económicas y políticas del mundo andino. Instituto de Estudios Peruanos. Abril. Lima, Perú.

Oberem, Udo.1981. El acceso a recursos naturales de diferentes ecologías en la Sierra ecuatoriana (XVI). En Contribuciones a la Etnohistoria Ecuatoriana, Editado por U. Oberem. Vol 20. IOA. Otavalo, Ecuador.

Salomon, Frank.1990. La politica vertical en las fronteras del Tawantinsuyo. Memoria, Volumen 1, No. 1, Marka, Quito.

Uhle, Max.1933. Estudio sobre las civilizaciones de Carchi e Imbabura. Informe al Señor Ministro de Educación Pública. Quito: Talleres Tipográficos Nacionales. Ecuador.

Uribe, María Victoria. 1977. Asentamientos prehispánicos en el altiplano de Ipiales, Colombia. En "Revista Colombiana de Antropología”. Instituto Colombiano de Cultura, Volumen XXI, Bogotá, Colombia.

Wittfogel, Karl.1966. Despotismo oriental. Ediciones Guadarrama, Madrid. España.

CONADE-INEHRI-ORSTOM. 1992. Funcionamiento del riego particular en los Andes Ecuatorianos. Recomendaciones para el plan de riego. Serie C3, Volumen Mira, Tomo 5. Ministerio de Agricultura y Ganadería, Abril. Quito, Ecuador. 